\title{
ELEMENTOS POSTCRANEANOS DE Cynognathus (SYNAPSIDA-CYNODONTIA) DEL TRIÁSICO INFERIOR DE LA PROVINCIA DE MENDOZA, ARGENTINA. CONSIDERACIONES SOBRE LA MORFOLOGÍA DEL HÚMERO EN CINODONTES
}

\author{
Fernando ABDALA \\ Laboratorio de Paleontología, Museu de Ciências e Tecnología, Pontificia \\ Universidade Católica de Rio Grande do Sul, Porto Alegre, Brasil. PIDBA, \\ Cátedra de Vertebrados, Facultad de Ciencias Naturales, Universidad Nacional \\ de Tucumán, Argentina.
}

\begin{abstract}
Abdala, F. 1999. Elementos postcraneanos de Cynognathus (Synapsida-Cynodontia) del Triásico Inferior de la provincia de Mendoza, Argentina. Consideraciones sobre la morfología del húmero en cinodontes. [Postcranial elements of Cynognathus (Synapsida-Cynodontia) from the Early Triassic of Mendoza province, Argentina. Considerations about the morphology of the humerus in cynodonts]. Revista Española de Paleontología, 14 (1), 13-24. ISSN 0213-6937.
\end{abstract}

\begin{abstract}
A vertebra and right humerus associated with a Cynognathus skull from the Early Triassic of Argentina are described. The vertebra is probably an anterior thoracic element, with the neural canal well developed, and the neural spine oriented $35^{\circ}$ posterior to the vertical. The humerus is long and expanded in both proximal and distal portions, and it lacks an ectepicondylar foramen. This bone is compared with the fragmentary humerus of the holotype of Cynognathus crateronotus Seeley, and with other complete bones tentatively assigned to the genus. These last elements show notable differences in the proportions of the proximal and distal portions, and in the longitudinal development of the deltopectoral crest in relation to the South American Cynognathus specimen, showing a morphology close to that of Diademodon. A comparison among groups of cynodonts is made. A general pattern for the cynodont humerus is presented, although two different morphologies for this bone are reported. The most remarkable features in one group is the comparatively higher mediolateral expansion on both the proximal and distal portions of the bone. Differences in the angulation between the deltopectoral crest and the axis passing through the head of the bone and the lesser tuberosity were also found. High angles are reported in basal cynodonts such as Procynosuchus, where it was considered as an adaptation for an aquatic environment, and also appears to be a response to larger size in traversodontids and chiniquodontids. Finally the general cynodont pattern is compared with that of different groups of mammals, from morganucodontids to marsupials (especially Didelphis).
\end{abstract}

Keywords: Cynodonts, Cynognathus, vertebra, humerus.

\section{RESUMEN}

Se describe una vértebra y un húmero derecho, asociados a un cráneo de Cynognathus del Triásico Inferior de Argentina. La vertebra posiblemente corresponde a un elemento torácico anterior, presentando el canal neural muy desarrollado y la espina neural inclinada aproximadamente $35^{\circ}$ con respecto a la vertical. El húmero es alargado y expandido en sus extremos, con la cabeza poco delimitada. No se observa el foramen ectepicondilar. Se compara el húmero con el elemento fragmentario del ejemplar tipo de Cynognathus crateronotus Seeley y con elementos completos asignados con dudas al género. Estos últimos muestran diferencias marcadas en las proporciones de las extremidades proximales y distales y en el desarrollo longitudinal de la cresta deltopectoral con respecto al ejemplar sudamericano. Se considera que estos últimos no corresponderían a Cynognathus, siendo mas próximos a la morfología del húmero de Diademodon. Se realiza una comparación de los húmeros de distintos grupos de cinodontes, presentándose un patrón general para el grupo. Sin embargo, se reportan dos morfologías diferentes de húmero, y algunas variantes presentes en distintos grupos de cinodontes. Finalmente se compara el patrón general del húmero de cinodontes con el de diferentes grupos de mamíferos, desde morganucodóntidos a marsupiales (particularmente Didelphis).

Palabras clave: Cinodontes, Cynognathus, vértebra, húmero. 


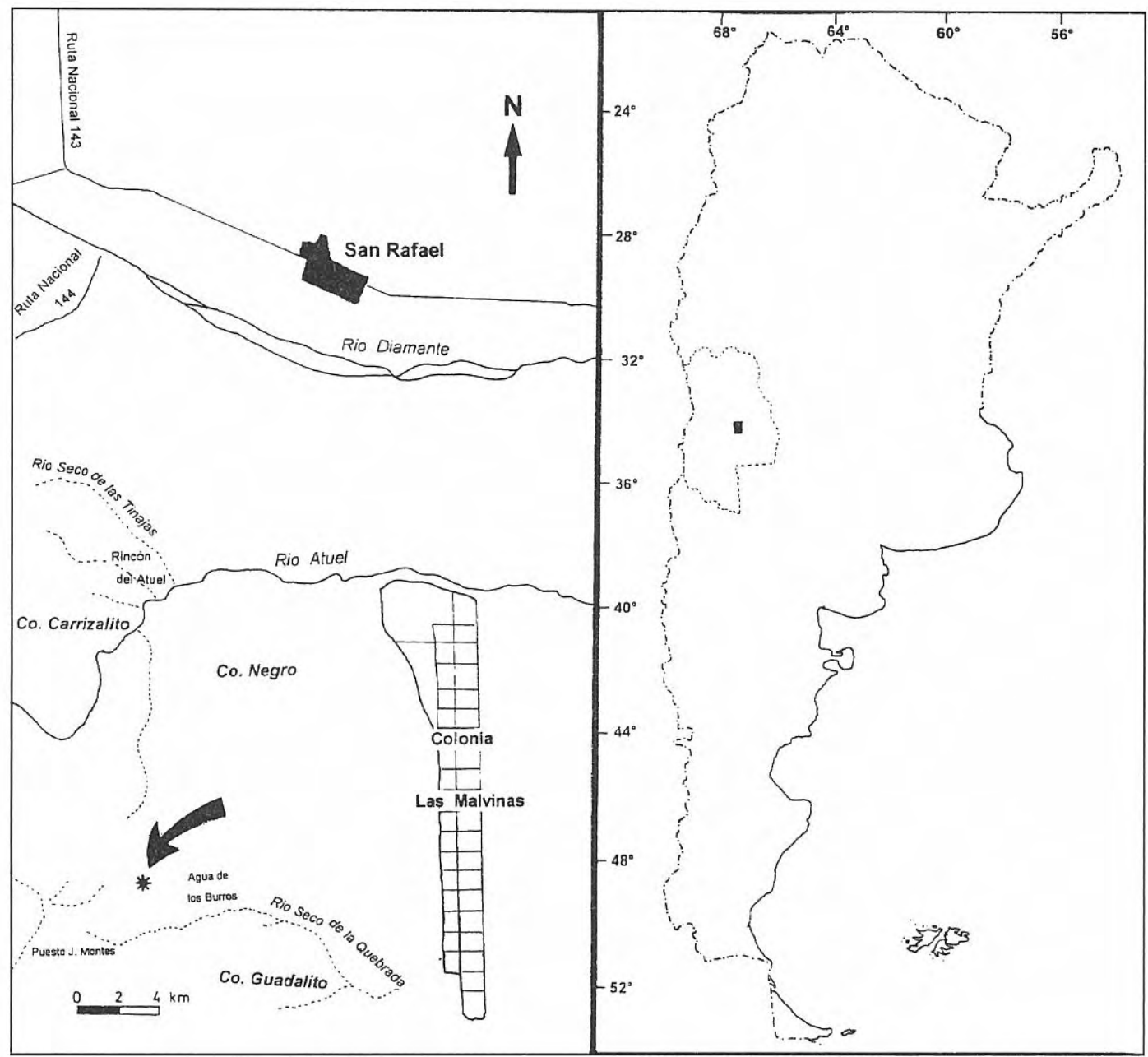

Figura 1. Mapa de ubicación del hallazgo (*).

\section{INTRODUCCIÓN}

Los cinognátidos representan los cinodontes carnívoros de mayor talla conocidos, alcanzando una longitud craneana de $36 \mathrm{~cm}$ y una longitud corporal superior a los $120 \mathrm{~cm}$. Se encuentran bien representados principalmente en Sudáfrica (Seeley, 1895b; Broili y Schröder, 1934, 1935; Brink, 1955, Kitching, 1977), pero en la década del 60 también fueron descubiertos en afloramientos del Triásico Inferior de Argentina (Bonaparte, 1969). Este hallazgo permitió reforzar la idea de una vinculación geográfica de los continentes del Gondwana durante el Triásico Inferior (Bonaparte, 1969).

El ejemplar estudiado se localizó en yacimientos distantes 800 m de Puesto Viejo (Gonzales Díaz, 1966), al oeste de la Colonia Las Malvinas en el Departamento de San Rafael, Provincia de Mendoza, Argentina (Figura 1) por una expedición de la Fundación Miguel Lillo, dirigida por el Dr. J. Bonaparte. El mismo procede de niveles correspondientes a la sección superior de la Formación Puesto Viejo (Gonzales Díaz, 1966, 1972), y se encontró asociado con restos de dicinodontes kannemeyéridos y de cinodontes traversodóntidos (Bonaparte, 1978). Esta formación es considerada, en principio, por la gran mayoría de autores como del
Triásico Inferior alto al correlacionarla con la fauna de la Zona de Cynognathus de Sudáfrica (Bonaparte, 1978; King, 1993; Anderson y Anderson, 1993). Sin embargo, Ochev y Shishkin (1989) interpretan a los depósitos de Zona de Cynognathus de Sudáfrica como del Triásico Medio bajo, y más recientemente Hancox y Rubidge (1996) encontraron en la parte superior de dicha zona dicinodontes de edad anisiana.

El ejemplar está representado por un cráneo con su mandíbula articulada, una vértebra y un húmero derecho. El cráneo fue originalmente descripto por Bonaparte (1969), quien lo nomina como Cynognathus minor. Recientemente Abdala (1996a) presentó una redescripción del mismo sinonimizándolo con Cynognathus crateronotus Seeley, 1895.

En esta contribución se presenta una descripción de los elementos postcraneanos asociados al cráneo. Anteriormente sólo el ejemplar holotipo de Cynognathus crateronotus había sido encontrado asociado con la parte proximal y distal del húmero, ambas mal conservadas (Seeley, 1895b). Por otra parte, elementos postcraneanos no asociados al cráneo fueron asignados con incertidumbre a los Cynognathidae (Watson, 1917; Jenkins, 1971). Por lo tanto, ésta constituye la primera contribución en donde la morfología de este elemento es 


\begin{tabular}{|l|l|}
\hline Longitud del centro & 1,3 \\
\hline Alto del centro & 1,6 \\
\hline Ancho del centro & 1,4 \\
\hline Ancho entre las apófisis transversas & 2,3 \\
\hline
\end{tabular}

Tabla 1. Dimensiones del elemento vertebral (en $\mathrm{cm}$ ).

conocida con certeza, permitiendo la comparación con los elementos postcraneanos tentativamente asignados a la familia. Adicionalmente, se compara el húmero de cinodontes no mamalianos con distintos grupos de mamíferos (i.e. mamaliaformes de Rowe, 1988), destacándose las principales diferencias encontradas entre los mismos.

\section{MATERIALES Y MÉTODOS}

El ejemplar de Cynognathus descripto está depositado en la colección Paleontología de Vertebrados Lillo (PVL 3859). Adicionalmente, se consultaron materiales de las siguientes instituciones: BMNH, Natural History Museum, Londres; BS, Bayerische Staatssammlung für Paläontologie und historische Geologie, Munich; GPIT, Institut und Museum für Geologie und Paläontologie, Universität Tübingen, Tübingen; MCZ, Museum of Comparative Zoology, Harvard University, Cambridge, MA; PVL, Colección Paleontología de Vertebrados Lillo, Universidad Nacional de Tucumán, Tucumán, Argentina; SAM, South African Museum, Cape Town; ZMO, Zoology Museum, Oxford University.

Los materiales comparativos consultados en las instituciones arriba mencionadas son los siguientes:

?Andescynodon: PVL 4424; PVL 4425; PVL 4426; PVL 4427: elementos postcraneanos aislados.

Belesodon: GPIT 40: Holotipo de Belesodon magnificus Huene (cráneo y numerosos elementos postcraneanos) descripto por Huene (1935-42).

Cynognathus: BMNH R2571: Holotipo de Cynognathus crateronotus (cráneo y gran parte del postcráneo) descripto por Seeley (1895b); BMNH R 3772a: húmero atribuido a Cynogntahus por Watson (1917).

Chiniquodon (sensu Abdala, 1996b): GPIT 1050: Holotipo de Chiniquodon theotonicus Huene (cráneo y húmero derecho) descripto por Huene (1935-42); MCZ 3781; MCZ 4002: esqueletos postcraneanos descriptos como Probelesodon lewisi Romer por Romer y Lewis (1973); PVL 3820: Numerosos elementos poscraneanos, incluyendo húmero y vertebras dorsales de Chiniquodon.

Diademodon: BMNH R 2579: húmero de ?Diademodon, descripto por Seeley (1895a); SAM 4002: diversos elementos postcraneanos, inclusive húmero, asociados a una mandíbula.

Exaeretodon: PVL 2467; PVL 2554: cráneo y elementos postcraneanos descriptos por Bonaparte (1963).

Luangwa: ZMO TSK 121: cráneo y elementos postcraneanos descriptos por Kemp (1980b).

Massetognathus: PVL 4613: cráneo y postcráneo.

Oligokyphus: numeroso material postcraneano la

\begin{tabular}{|l|l|}
\hline Longitud (desde la cabeza al ectepicóndilo) & 13,5 \\
\hline Ancho distal (ectepicóndilo-entepicóndilo) & 4,5 \\
\hline Ancho proximal & 4,4 \\
\hline Longitud de la cresta deltopectoral & 6,6 \\
\hline
\end{tabular}

Tabla 2. Dimensiones del húmero (en $\mathrm{cm})$.

mayoría del mismo descripto por Kühne (1956).

Probainognathus: PVL 4677: Cráneo con mandíbula en oclusión y gran parte del postcráneo; PVL 4724; PVL 4725: elementos postcraneanos.

Procynosuchus: ZMO TSK 34: cráneo y postcraneo descripto por Kemp (1979; 1980a).

Thrinaxodon: BS 1934 VIII 506: cráneo, placas costales, húmeros, clavícula e interclavícula.

También se consultaron restos de mamíferos actuales, especialmente marsupiales, pertenecientes a las colecciones PVL, CML (Colección de Mamíferos Lillo) y la Colección Rusconi, de la Facultad de Ciencias Naturales de la Universidad Nacional de Tucumán.

La vértebra descripta fue comparada principalmente con el holotipo de Cynognathus crateronotus (BMNH R2571), aunque algunos rasgos fueron también considerados de cinodontes traversodóntidos y tritilodóntidos.

El húmero fue comparado con los elementos fragmentarios del holotipo de C. crateronotus (BMNH R2571), asi como con los húmeros asignados a cinognátidos no asociados con el cráneo (Watson, 1917; Jenkins, 1971).

Tanto los materiales del ejemplar de Cynognathus acá presentados como la mayoría del material comparativo fueron medidos mayormente en forma directa, aunque en algunos casos las dimensiones fueron obtenidas a partir de la literatura.

Las mediciones de la vértebra y el húmero del ejemplar son presentadas en las Tablas 1 y 2 , respectivamente.

En la Tabla 3 se considera la angulación entre la cresta

\begin{tabular}{|c|c|c|}
\hline Taxón & Angulación & $\begin{array}{c}\text { Longitud del } \\
\text { húmero } \\
(\mathbf{e n ~ c m )}\end{array}$ \\
\hline Massetognathus & $90^{\circ}$ & 5,4 \\
\hline ?Andescynodon & $90^{\circ}$ & 4,4 \\
\hline Pascualgnathus & $90^{\circ}$ & 5,7 \\
\hline Luangwa & $100^{\circ}$ & 8,7 \\
\hline Oligokyphus & $100^{\circ}$ & 4,1 \\
\hline ?Cynognathus (?Diademodon) & $110^{\circ}$ & 10,7 \\
\hline Cynognathus & $120^{\circ}$ & 13,5 \\
\hline Chiniquodon & $125^{\circ}$ & 7,4 \\
\hline Belesodon & $150^{\circ}$ & 15,0 \\
\hline Thrinaxodon & $135^{\circ}$ & 3,5 \\
\hline Probainognathus & $135^{\circ}$ & 3,8 \\
\hline Procynosuchus & $145^{\circ}$ & 6,7 \\
\hline Exaeretodon & $150^{\circ}$ & 17,5 \\
\hline
\end{tabular}

Tabla 3. Angulación entre la cresta deltopectoral en diferentes grupos de cinodontes. La misma se observa en el extremo proximal del húmero y se forma entre la cresta y el borde que se extiende entre la cabeza humeral y la tuberosidad menor. 


\begin{tabular}{|c|c|c|c|c|}
\hline TAXON & MATERIAL & AP & AD & LC \\
\hline Procynosuchus & ZMO TSK 34 & $36 \%$ & $49 \%$ & $52 \%$ \\
\hline Thrinaxodon & Jenkins (1971) & $32 \%$ & $49 \%$ & $52 \%$ \\
\hline Cynognathus & PVL 3859 & $33 \%$ & $39 \%$ & $49 \%$ \\
\hline Cricodon & Crompton (1955) & $30 \%$ & $42 \%$ & $50 \%$ \\
\hline ?Cynognathus (?Diademodon) & Jenkins (1971) & $36 \%$ & $46 \%$ & $55 \%$ \\
\hline ?Cynognathus & BMNH R 3772a & $42 \%$ & $52 \%$ & $62 \%$ \\
\hline ?Diademodon & BMNH R 2579 & $37 \%$ & $45 \%$ & $57 \%$ \\
\hline Diademodon & SAM 4002 & $35 \%$ & $50 \%$ & $50 \%$ \\
\hline Pascualgnathus & Bonaparte (1966) & $32 \%$ & $46 \%$ & $58 \%$ \\
\hline ?Andescynodon & PVL 4424; PVL 4425; & $27 \%-32 \%$ & $41 \%-50 \%$ & $54 \%-61 \%$ \\
& PVL 4426; PVL 4427 & & & \\
\hline Massetognathus & PVL 4613; PVL sn & $27 \%-30 \%$ & $38 \%-40 \%$ & $52 \%$ \\
\hline Luangwa & ZMO TSK 121 & $32 \%$ & $41 \%$ & $58 \%$ \\
\hline Exaeretodon & PVL 2467; PVL sn & $40 \%-41 \%$ & $49 \%-57 \%$ & $52 \%-60 \%$ \\
\hline Probainognathus & PVL 4677; PVL sn & $24 \%-26 \%$ & $34 \%-37 \%$ & $45 \%-50 \%$ \\
\hline Chiniquodon & MCZ 4002; PVL 3820; GPIT 1050 & $38 \%-41 \%$ & $58 \%-63 \%$ & $54 \%-57 \%$ \\
\hline Belesodon & GPIT 40 & $39 \%$ & $59 \%$ & $59 \%$ \\
\hline Kayentatherium & Sues (1983) & $41 \%$ & $54 \%$ & - \\
\hline Oligokyphus & Kuhne (1956) & $29 \%$ & $46 \%$ & - \\
\hline Eozostrodon & Jenkins y Parrington (1976) & $26 \%$ & $30 \%$ & $44 \%$ \\
\hline Gobiconodon & Jenkins y Schaff (1988) & - & $43 \%$ & $53 \%$ \\
\hline ?Lambdopsalis & Kielan-Jaworowska y Qi (1990) & $31 \%-34 \%$ & $41 \%-44 \%$ & $66 \%$ \\
\hline cf. Bulganbaatar & Sereno y McKenna (1995) & $23 \%$ & $22 \%$ & $54 \%$ \\
\hline Vincelestes & Rougier (1993) & $22 \%-23 \%$ & $36 \%$ & $42 \%$ \\
\hline Didelphis & CML (colección osteológica) & $23 \%$ & $29 \%$ & $64 \%$ \\
\hline Macropus & CML(colección osteológica) & $21 \%$ & $28 \%$ & $61 \%$ \\
\hline
\end{tabular}

Tabla 4. Indices AP (ancho proximal/longitud del humero x 100); AD (ancho distal/longitud del húmero x 100) y LC (longitud desde la cabeza al borde distal de la cresta deltopectoral/longitud del húmero x 100). AP medido desde la tuberosidad menor hasta el borde medial de la cabeza; AD medido desde el ectepicóndilo al entepicóndilo.

deltopectoral y el eje que se extiende entre la cabeza humeral y la tuberosidad menor. Dicha angulación es observable en una vista proximal del elemento (ver figura 4a).

En la Tabla 4 se presentan las relaciones porcentuales entre el ancho proximal/longitud del humero (AP), el ancho distal/longitud del húmero (AD) y la longitud desde la cabeza humeral al borde distal de la cresta deltopectoral/longitud del húmero (LC). El ancho proximal es medido desde la tuberosidad menor hasta el borde medial de la cabeza. El último punto es elegido preferentemente a la tuberosidad mayor, difícil de distinguir en muchos de los elementos observados. El ancho distal se mide desde el ectepicóndilo al entepicóndilo.

\section{DESCRIPCIÓN}

VÉRTEBRA (Figs. 2 y 3 )

Este elemento corresponde a una vertebra torácica, probablemente anterior. No conserva las zigapófisis y la espina neural está incompleta. El centro anficélico es cilíndrico, de altura, largo y ancho casi similares (ver Tabla 1). El canal neural está muy desarrollado, siendo en sección algo menor que el centro. Los procesos transversos se encuentran a nivel de la parte media del canal. Dorsalmente al mismo se encuentran las prezigapófisis, dirigidas anteriormente e incompletas, no habiéndose conservado las facetas de articulación. La espina neural es robusta en su base y si bien está incompleta, se puede determinar una inclinación posterior de aproximadamente $35^{\circ}$ con respecto al eje vertical.

\section{Comparación con otros cinodontes}

El ejemplar holotipo de $C$. crateronotus (BMNH R 2571) posee gran parte de la columna vertebral conservada (Seeley, 1895b), presentando 15 vértebras torácicas (Jenkins, 1971, pag. 68). El centro vertebral del ejemplar aquí descripto muestra similitud en sus proporciones con los centros torácicos anteriores del mencionado espécimen. Para los mismos, Seeley (1895b) menciona una longitud de $2,9 \mathrm{~cm}$, el ancho en la articulación intervertebral de $3,8 \mathrm{~cm}$ y una altura de 3,8 $\mathrm{cm}$. En las dorsales posteriores, la longitud aumenta hasta los 3,2 cm (Jenkins, 1971) mientras que la altura se reduce a $2,8 \mathrm{~cm}$ (Seeley, 1895b). Como rasgo diferencial, el ejemplar sudafricano presenta las vértebras torácicas con sus espinas neurales dirigidas verticalmente o débilmente inclinadas hacia atrás (Seeley, 1895b). Una inclinación en la espina neural de vértebras torácicas 


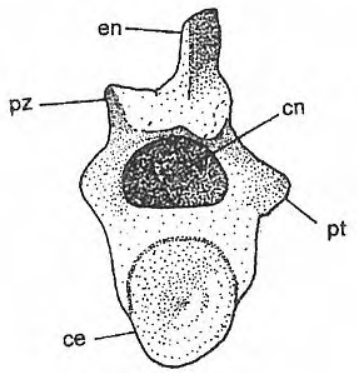

a

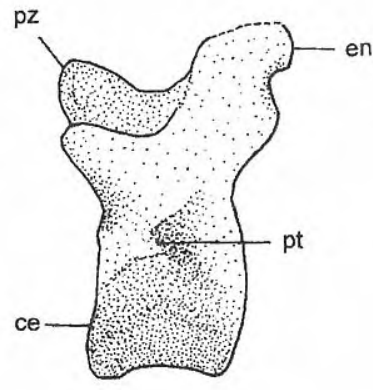

b

Figura 2. Vértebra dorsal de Cynognathus. a. Vista anterior y b. Vista lateral. Abreviaturas: ce, centro; cn, canal neural; en, espina neural; pt, proceso transverso; pz, prezigapófisis. Escala $1 \mathrm{~cm}$.

(=dorsales anteriores) de $30^{\circ}$ ha sido señalada para Massetognathus (Jenkins, 1970a) y se observa asimismo en Exaeretodon (Bonaparte, 1963, fig. 1). Un mayor grado de inclinación de las espinas torácicas caracteriza a tritilodóntidos (Sun y Li, 1985; Kuhne, 1956, Sues, 1983) y morganucodóntidos (Jenkins y Parrington, 1976), donde puede alcanzar hasta $60^{\circ}$.

\section{HÚMERO (Figs. 4 y 5)}

Como es característico en cinodontes, el húmero es robusto, alargado y expandido en sus extremos proximal y distal. No obstante, este elemento es más grácil que en la mayoría de los cinodontes conocidos (Figs. 6 y 7). Las medidas de este elemento se presentan en la Tabla 2.

La cabeza humeral es levemente convexa, sobresaliendo apenas hacia arriba. La tuberosidad mayor está bien desarrollada, aun cuando su conservación no es completa. La tuberosidad menor es un área redondeada y algo expandida medialmente. El canal bicipital, dispuesto entre ambas tuberosidades es amplio.

La cresta deltopectoral, aunque incompleta en su extremo libre se presenta muy desarrollada y se extiende hasta la mitad del hueso. El ángulo entre la cresta y el eje que pasa entre la tuberosidades mayor y menor (Figura 3a) es de aproximadamente $120^{\circ}$. Donde se ha conservado, el borde libre de la cresta muestra estriaciones marcadas en la cara lateral que indican una fuerte inserción del músculo deltoides (Romer, 1922; Jenkins, 1971; Kemp, 1980a, b). En su cara lateral, la cresta presenta una depresión superficial, apenas marcada, interpretada por Jenkins (1971) como posible área de inserción para el músculo braquialis. La misma se encuentra limitada por una cresta robusta, probablemente para la inserción del teres minor.

El extremo distal del húmero presenta el entepicóndilo incompleto. El foramen ectepicondilar está ausente, mientras que el entepicondilar, como es típico en cinodontes, es oval y alargado.

Dorsalmente la porción distal del húmero muestra una depresión triangular bien marcada, probablemente correspondiente a un esbozo de la fosa olecraneana. En vista ventral, el capitulum (cóndilo lateral) es una protuberancia bulbosa, notablemente más desarrollada y más extendida ventralmente que el cóndilo ulnar.
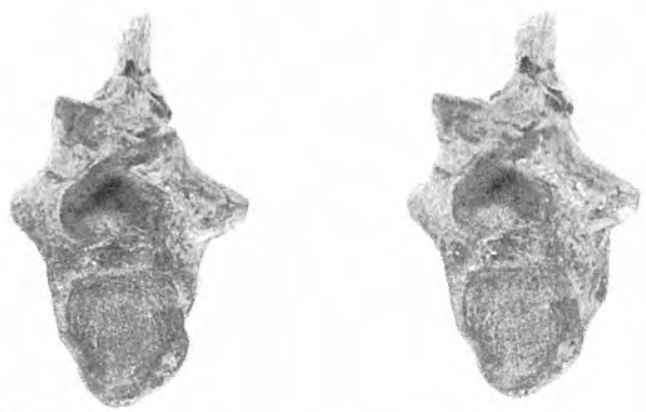

2
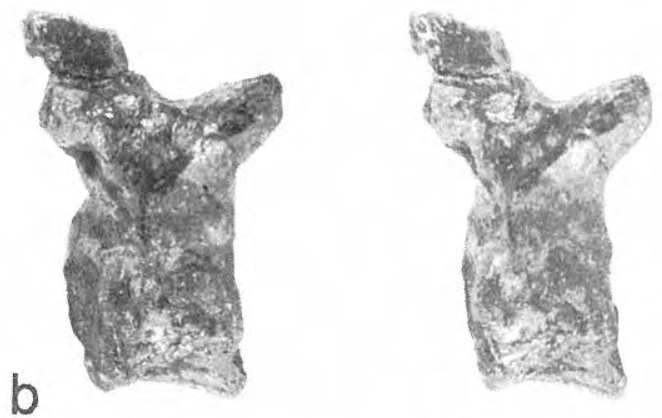

Figura 3. Estereofotografía de la vértebra dorsal de Cynognathus en a. Vista anterior y b. Vista lateral. Escala $1 \mathrm{~cm}$.

\section{Comparación con otros ejemplares asignados a cinognátidos}

Los únicos elementos humerales asignado sin dudas a Cynognathus fueron encontrados asociados al ejemplar tipo descripto por Seeley (1895b), correspondiendo a las porciones proximal y distal del húmero derecho (BMNH R2571). La porción distal está mal conservada no aportando datos comparativos de utilidad. La porción proximal presenta marcadas similitudes con el ejemplar aquí descripto. La cabeza está algo más proyectada de la diáfisis respecto al ejemplar sudamericano, pero menos que en los restantes cinodontes. La porción de la cresta deltopectoral visible no presenta el extremo libre muy separado de la diáfisis. Si bien Seeley (1895b, pág. 95) menciona una angulación de alrededor de $100^{\circ}$ con el eje de la diáfisis, la misma es mayor, correspondiendo aproximadamente a $120^{\circ}$ (Abdala, ob. pers.)

Otro húmero completo (BMNH 3772a) asignado por Watson (1917) a cinognátidos presenta algunas particularidades que merecen destacarse. El fragmento proximal de húmero del ejemplar tipo de $C$. crateronotus (BMNH R2571) corresponde a uno de los especimenes de mayor talla craneana en la Familia Cynognathidae y por extensión en los cinodontes carnívoros. Sin embargo, el húmero descripto por Watson (1917) presenta una longitud de $26,1 \mathrm{~cm}$ y su extremo proximal marcadamente mayor que el del especimen BMNH R2571, por lo cual este ejemplar, de ser cinognátido, correspondería a un individuo de mayor talla que los conocidos actualmente. Considerando los valores presentados en la Tabla 4, tenemos que tanto el ejemplar estudiado por Watson (1917) como el de ?Cynognathus 

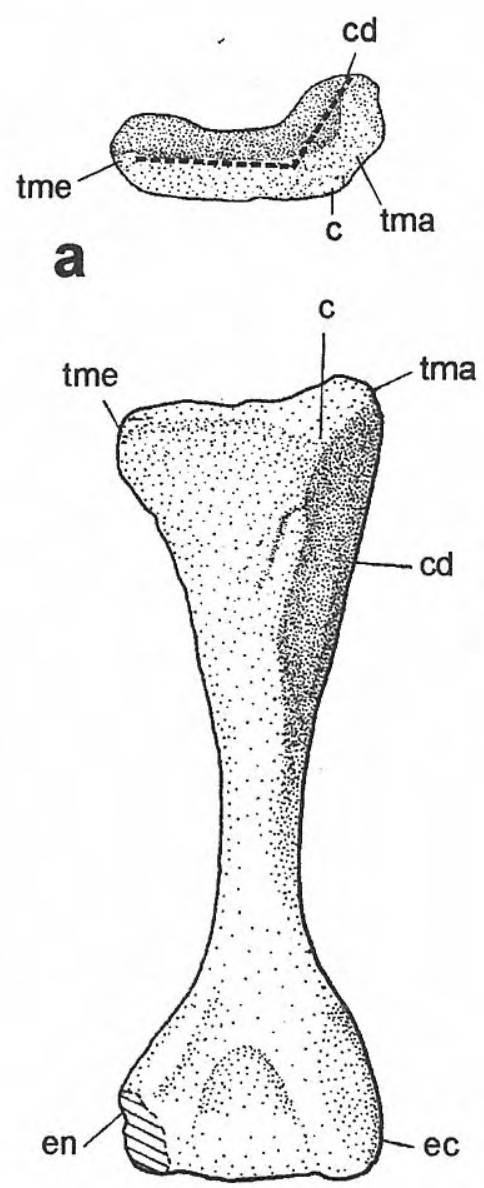

b

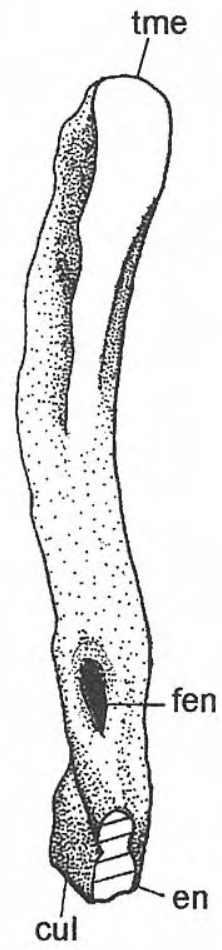

C
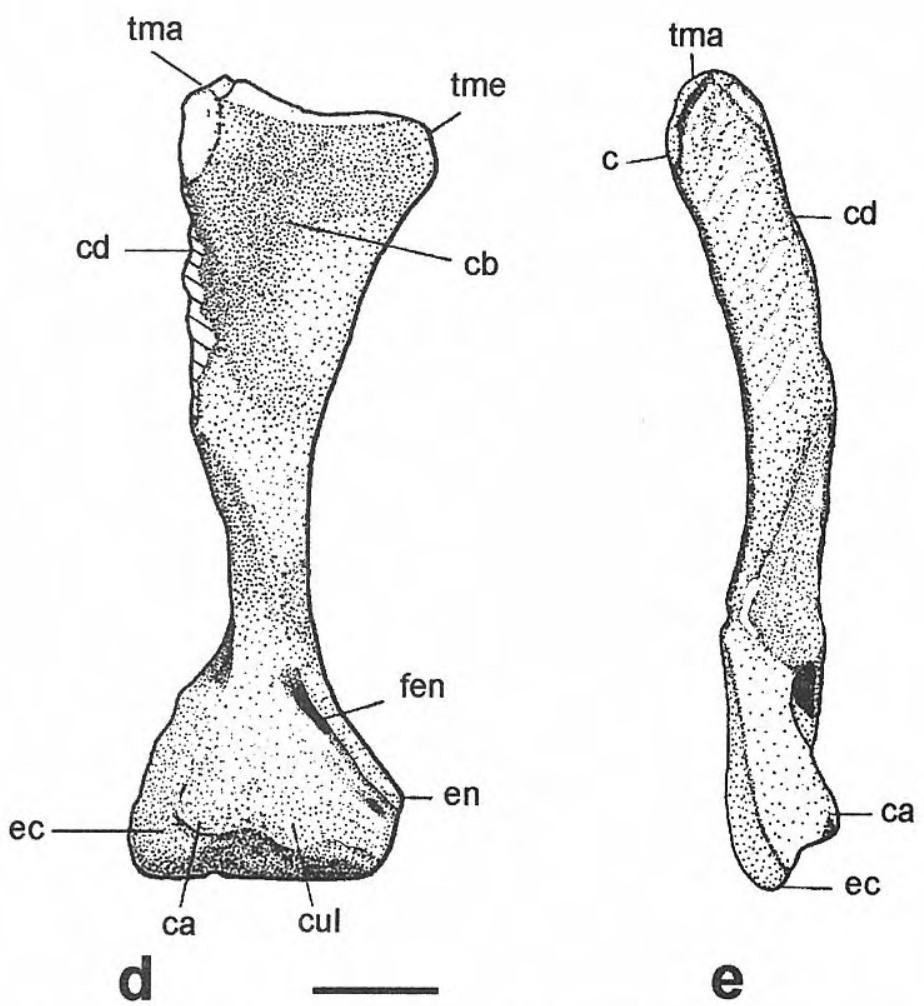

e

Figura 4. Húmero derecho de Cynognathus. a. Vista proximal, b. Dorsal, c. Medial, d. Ventral, e. Lateral. Abreviaturas: c, cabeza humeral; ca, capitulum; cb, canal bicipital; cd, cresta deltopectoral; cul, cóndilo ulnar; ec, ectepicóndilo; en, entepicóndilo; fen, foramen entepicondilar; tma, tuberosidad mayor; tme, tuberosidad menor. Escala $2 \mathrm{~cm}$.

(?Diademodon) descripto por Jenkins (1971), presentan diferencias proporcionales marcadas con el ejemplar aquí descripto. La diáfisis del especimen sudamericano es más grácil y alargada y con la cabeza notablemente menos expandida. Adicionalmente, hay un mayor desarrollo longitudinal de la cresta deltopectoral en el ejemplar descripto por Watson (1917), donde esta estructura presenta el mayor desarrollo axial observado en cinodontes (ver Tabla 4). El extremo distal está muy ensanchado en los especímenes descriptos por Watson (1917) y Jenkins (1971), mientras que en el ejemplar sudamericano, si bien dicho extremo no está completo, su ensanchamiento no sería tan marcado. Finalmente, la mayor similitud en proporciones se observa entre el extremo proximal del ejemplar aquí descripto con el de Jenkins (1971), correspondiendo al $33 \%$ y al $36 \%$, respectivamente.

A las diferencias que presenta el ejemplar sudamericano con los ejemplares arriba señalados debe agregarse que los elementos descriptos por Watson (1917) y Jenkins (1971) poseen una marcada similitud con el ejemplar SAM 4002, correspondiente a Diademodon. Las similitudes son significativas particularmente en el grado de proyección de la cabeza humeral, el desarrollo relativo de la extremidades proximal y distal y la morfología de la cresta deltopectoral (aunque no en su longitud, ver Tabla 4), mostrando una angulación marcada en el extremo distal de la misma, similar en los tres elementos. Esta situación indica que los húmeros asignados por Watson (1917) a cinognátidos y por Jenkins (1971) a ?Cynognathus (?Diademodon) no presentan la morfología característica de cinognátidos, siendo asignables con mayor certeza a diademodóntidos.

\section{MORFOLOGÍA DEL HÚMERO EN CINODONTES}

El húmero en los cinodontes muestra una serie de rasgos comunes que permiten reconocer un patrón característico. Los caracteres mas destacables son la cresta deltopectoral claramente separada de la diáfisis, la tuberosidad menor constituyendo una estructura bulbosa; la tuberosidad mayor no diferenciada claramente de la cabeza humeral; el canal bicipital amplio lateromedial- 

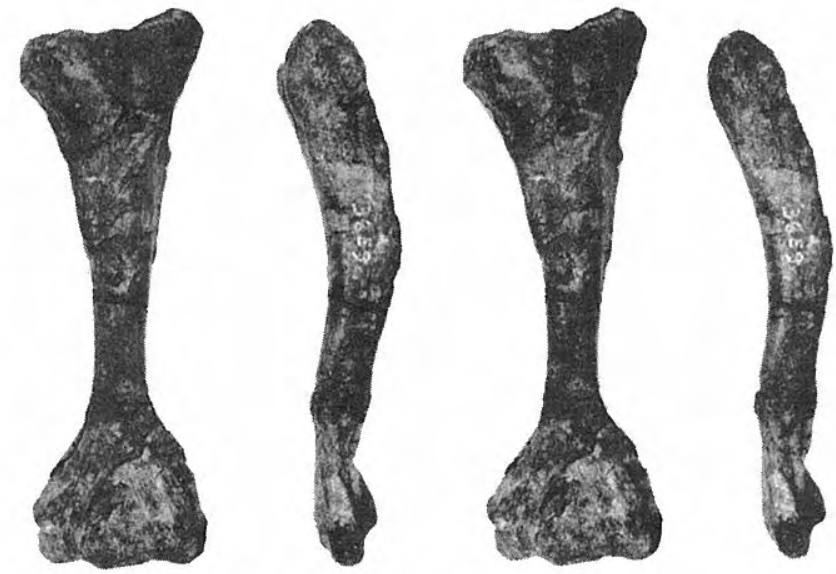

a
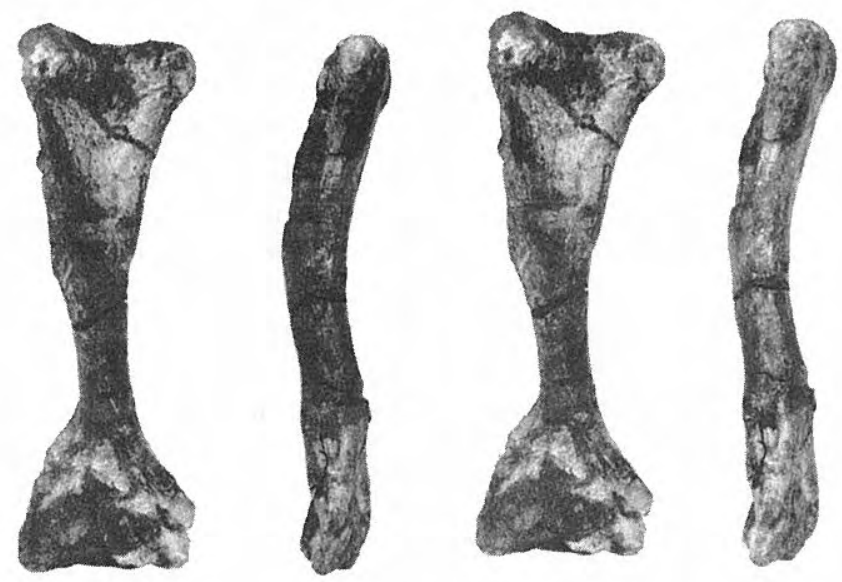

b
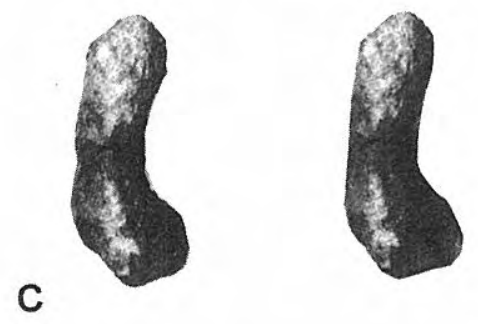

Figura 5. Estereofotografías de a. Vista dorsal y lateral, b. Vista ventral y medial y c. Vista proximal del húmero de Cynognathus. Escala $2 \mathrm{~cm}$.

mente y los extremos proximal y distal del hueso lateromedialmente expandidos. También debe mencionarse la presencia común, por lo general muy notable, de la protuberancia para el teres major en la cara medial del hueso.

Sin embargo, pueden distinguirse al menos dos tipos de húmeros (Figs. 6 y 7). Uno de ellos presenta la diáfisis más bien corta y robusta, con ambas extremida-des generalmente muy desarrolladas lateromedialmente. Este patrón fue reconocido por Bonaparte (1963), quien lo consideró como de "aspecto primitivo" por su semejanza al de algunos gorgonopsios y dicinodontes (e.g. Dicynodon y Kannemeyeria, Watson, 1917, Ischigualastia, Cox, 1965). Este tipo de húmero está presente en dos familias de cinodontes: en el traversodóntido Exaeretodon (Bonaparte, 1963) y en los chiniquodóntidos.

El segundo tipo de húmero muestra la diáfisis más esbelta, siendo la relación con los extremos del húmero variable. Por lo general, el extremo proximal es poco expandido, aun cuando en este grupo también hay formas con una angulación marcada entre la cresta deltopectoral y el eje de la diáfisis (ver abajo). La mayor parte de los cinodontes presentan este tipo de morfología (Figs. 6 y 7), entre otros, Probainognathus (Abdala, 1996b), Pascualgnathus (Bonaparte, 1966) Massetognathus (Jenkins, 1970a), ?Andescynodon, Oligokyphus (Kuhne, 1956), Bienotheroides (Sun y Li, 1985) y Kayentatherium (Sues, com. pers., 1997). Thrinaxodon presenta el húmero con una condición mas próxima a la segunda mencionada, pero con su extremo distal muy ensanchado (Fig. 7).

La angulación de la cresta deltopectoral con respecto al eje que pasa entre la cabeza y la tuberosidad menor presenta cierta variación en cinodontes. En ?Andescynodon, Pascualgnathus (Bonaparte, 1966) y Massetognathus, la misma es cercana a los $90^{\circ}$; Luangwa presenta aproximadamente $100^{\circ}$ (Kemp, 1980b); Cynognathus, $120^{\circ}$, Probainognathus, $135^{\circ} \mathrm{y}$, finalmente, formas con la cresta muy abierta son Procynosuchus $\left(145^{\circ}\right)$, Exaeretodon $\left(150^{\circ}\right)$ y Belesodon $\left(150^{\circ}\right)$. Internamente diversos grupos de cinodontes muestran variación en este rasgo. En los chiniquodóntidos de menor talla, la cresta deltopectoral está notablemente más cerrada que en formas mayores y la misma situación se verifica en los traversodóntidos (Tabla 3). Es así que la presencia de la cresta deltopectoral abierta, más que un rasgo plesiomórfico en cinodontes (Bonaparte, 1963), fue interpretada, por un lado, como una adaptación para la vida en el medio acuático en el caso de Procynosuchus (Kemp, 1980a), y además se presenta independientemente en traversodóntidos y chiniquodóntidos de grandes tallas (Fig. 8). La existencia de esta morfología de cresta deltopectoral en cinodontes de gran porte se interpreta como posiblemente relacionada con una mayor importancia de los músculos pectorales para el sostén y elevación del cuerpo del sustrato en animales de gran talla que no presentaban aun las extremidades anteriores posicionadas debajo del cuerpo. La función de los músculos pectorales como aductores de la extremidad y para elevar el cuerpo del sustrato es más importante en tetrápodos basales que presentan postura tipo "sprawling" (Walker, 1987), mientras que en mamíferos, dichos músculos son principalmente retractores y poco importantes en el sostén corporal, que es principalmente llevado a cabo por la modificación en la posición de las extremidades (Hildebrand, 1988). Como una alternativa a la funcionalidad arriba mencionada, debe señalarse que la presencia de cresta deltopectoral muy desarrollada y algo abierta con respecto a la diáfisis se conoce en animales con habitos cavadores (Hildebrand, 1988) y también es característico de monotremas (e. g. Tachyglossus), formas 


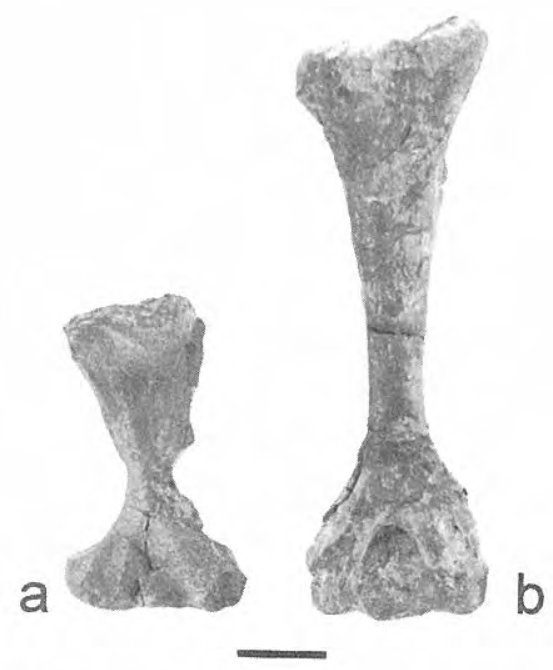

Figura 6. Vista dorsal de los húmeros de a. Chiniquodon y b. Cynognathus. Escala $2 \mathrm{~cm}$.

donde el húmero tiende a presentar como principal movilidad la rotación alrededor de su eje (Jenkins, 1970b).

Gran parte de los cinodontes presentan la cabeza humeral bien esbozada, sobresaliendo de la diáfisis a la que se une por medio de un cuello incipiente (Fig. 7). Este es el caso de la mayoría de traversodóntidos, tritilodóntidos, chiniquodóntidos y probainognátidos. Mucho menos marcado es el desarrollo de la misma en Procynosuchus (Kemp, 1980a, fig. 9) y en Cynognathus, donde la cabeza está apenas esbozada. En los representantes más robustos de los chiniquodóntidos, traversodóntidos y tritilodóntidos, la cabeza se encuentra más extendida en el lado dorsal (posterior) del húmero.

Con respecto al foramen ectepicondilar, su presencia es variable entre los cinodontes. El especimen de Cynognathus aquí descripto no lo presenta, al igual que Probainognathus (PVL 4677) y todos los tritilodóntidos donde el húmero es conocido (Young, 1947; Kuhne, 1956; Sues, 1983; Sun y Li, 1985). Los restantes cinodontes presentan foramen ectepicondilar. Debe destacarse que esta estructura resulta particularmente difícil de visualizar debido a su mala conservación en ?Andescynodon, donde de cinco elementos solo en uno pudo observarse. La historia de este foramen en los sinápsidos es compleja, aunque su ausencia probablemente represente una sinapomorfía de cinodontes avanzados y mamíferos. Edaphosaurus entre los pelicosaurios (Romer, 1922), los anomodontes, Taphinocephalus entre los dinocéfalos, algunos bauriamorfos (Broom, 1932, 1948) y la mayoría de los cinodontes presentan dicho foramen. Por otro lado, su ausencia se registra en los pelicosaurios (Romer, 1922), dinocéfalos, terocéfalos, algunos cinodontes avanzados y en mamíferos (Broom, 1948; Jenkins y Parrington, 1976; Kemp, 1986).

En chiniquodóntidos y en Cynognathus, el entepicóndilo es una proyección delgada, mientras que el ectepicóndilo, próximo al cual se encuentra el capítulo, es una protuberancia más robusta. En Massetognathus y ?Andescynodon, el entepicóndilo aparenta ser más robusto que en chiniquodontes. Jenkins (1971) menciona que en cinodontes de mayor talla, el ectepicóndilo está más desarrollado que el entepicóndilo, interpretándolo como una necesidad para la transferencia del peso corporal al radio. Para formas de menor tamaño, como por ejemplo Thrinaxodon, donde el peso no es un factor crítico, Jenkins (1971) destaca que el entepicóndilo muestra un desarrollo mayor. Sin embargo, aun cuando en formas pequeñas, como terocéfalos regisauridos (Kemp, 1986, fig.6F), Probainognathus (Jenkins, 1973, figs. 9 y 10) y Oligokyphus (Kuhne, 1956, text-fig. 54), se observa una disminución del tamaño del ectepicóndilo, al compararlos con formas de mayor talla, el desarrollo proporcional de ambos epicóndilos es similar, o bien el ectepicóndilo permanece como el elemento predominante de la extremidad distal. La presencia de epicóndilos expandidos en la extremidad distal del húmero de cinodontes ha sido relacionada con la presencia de músculos extensores y especialmente flexores robustos que aun deben ejercer una gran fuerza elevadora, probablemente por la posición inclinada del antebrazo, mientras que en mamíferos el antebrazo tendría una posición mas vertical y la función de sustentación sería mayormente asumida por el radio y en menor medida por los músculos del antebrazo (Romer, 1922).

Algunos rasgos diferenciales pueden señalarse en particular para el húmero de Probainognathus (Figura 7). La diáfisis es marcadamente gracil y alargada, y la cresta medial está muy extendida, mostrando una extensión casi similar a la deltopectoral. Tanto el extremo proximal como el distal de Probainognathus presentan la menor expansión en cinodontes, siendo proporcionalmente cercanos a los de Eozostrodon y Vincelestes (Tabla 4). El foramen entepicondilar es comparativamente más cerrado en su eje menor que en los restantes cinodontes y no presentan la proyección del entepicóndilo.

\section{COMPARACIÓN DEL HÚMERO DE CINODONTES NO MAMALIANOS Y DE MAMÍFEROS}

En este punto se intentan reconocer algunas diferencias entre la morfología general del húmero de cinodontes y grupos de mamíferos que representan distintas etapas filogenéticas que median entre los mamaliaformes y los teria (Rowe, 1988). Asimismo se reportan diferencias con respecto a los marsupiales, particularmente Didelphis, dado que presentan el húmero con un mayor grado de semajanza al observado en cinodontes y en algunos grupos de mamíferos no terios. Contrariamente este elemento en los monotremas muestra notables diferencias, interpretadas como modificaciones ligadas a su particular modo de vida (Lessertisseur y Saban, 1967; Jenkins, 1971).

Una de las diferencias más notables es la cabeza humeral ovoide, muy desarrollada, dirigida dorsalmente en los mamaliaformes y con un cuello particularmente notable en multituberculados (Kielan-Jaworowska y Qi, 1990; Sereno y McKenna, 1995), el triconodonte 


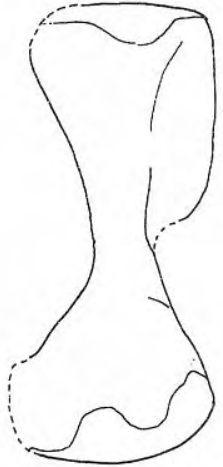

Procynosuchus

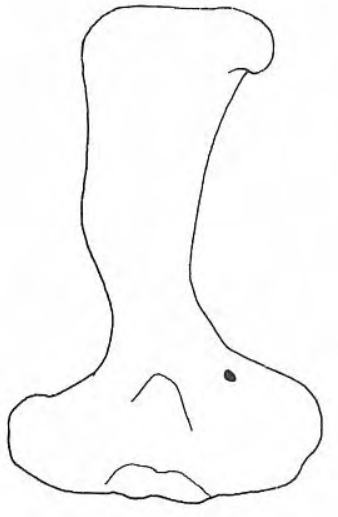

Chiniquodon

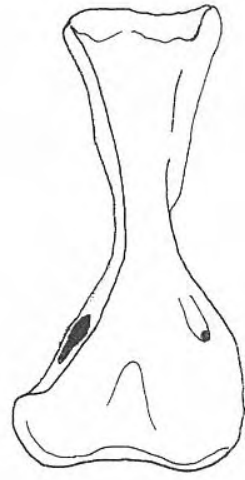

Thrinaxodon

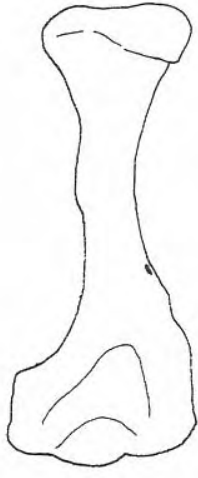

Luangwa

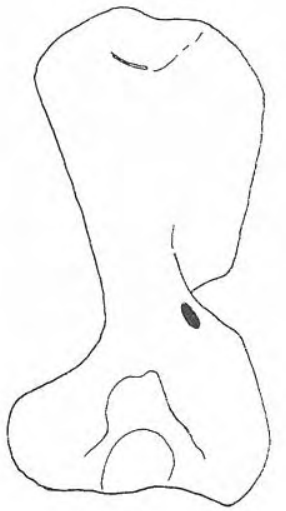

Exaeretodon

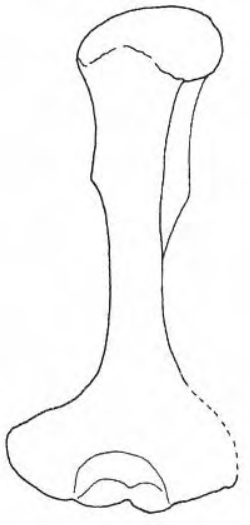

Oligokyphus
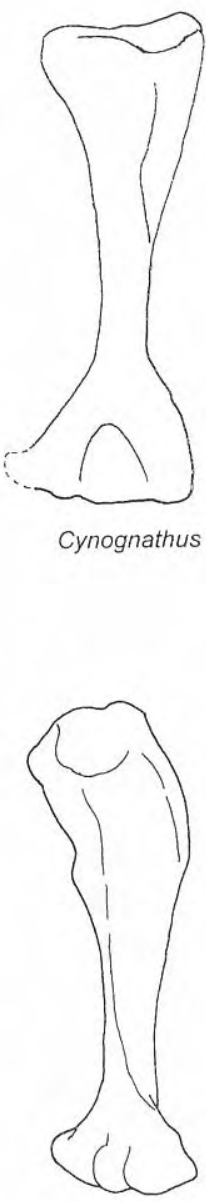

Eozostrodon

Figura 7. Vista dorsal de húmeros de diferentes cinodontes no mamalianos. Procynosuchus (de Kemp, 1980a); Thrinaxodon (de Jenkins, 1971); Luangwa (de Kemp, 1980b); Exaeretodon (de Bonaparte, 1963); Chiniquodon (de Romer y Lewis, 1973); Oligokyphus (de Kuhne, 1956); Eozostrodon (de Jenkins y Parrington, 1976). No a escala.

Gobiconodon (Jenkins y Schaff, 1988) y el terio prototribosfénico Vincelestes (Rougier, 1993). Didelphis presenta la cabeza del húmero redondeada, con alguna semejanza a la de Eozostrodon (Jenkins y Parrington, 1976) y especialmente a la del multituberculado cf. Bulganbaatar (Sereno y McKenna, 1995).

Se observan tendencias al estrechamiento de la parte proximal del húmero (Tabla 4; AP) y a la diferenciación de las tuberosidades, aun cuando por lo general la tuberosidad mayor permanece algo confluyente con la cabeza (Jenkins y Parrington, 1976; Kielan-Jaworowska y Qi, 1990). El canal bicipital en morganucodóntidos (Jenkins y Parrington, 1976), Gobiconodon (Jenkins y Schaff, 1988) y Vincelestes (Rougier, 1993) es, al igual que en cinodontes, amplio y superficial. En el multituberculado ?Lambdopsalis esta estructura se hace algo mas profunda en el lado de la tuberosidad mayor (Kielan-Jaworowska y Qi, 1990), mientras que en cf. Bulganbaatar (Sereno y McKenna, 1995) el canal bicipital es marcadamente más estrecho, siendo practicamente similar al observado en Didelphis.

La cresta deltopectoral es poco extensa en los morganucodóntidos y en Vincelestes, donde no sobrepasa la mitad de la longitud del húmero (Tabla 4, LC).
Contrariamente, en Gobiconodon (Jenkins y Schaff, 1988), ?Lambdopsalis (Kielan-Jaworowska y Qi, 1990), cf. Bulganbaatar (Sereno y McKenna, 1995, fig. 3e), en el simetrodonte Zhangheotherium (Hu et al., 1997, fig. 1) y en Didelphis ésta se extiende más allá de la mitad del hueso, condición observada en la mayoría de los cinodontes.

Al igual que en el extremo proximal del húmero, existe una tendencia a la reducción del ancho de la extremidad distal con las proporciones menores observadas en Eozostodon, en el multituberculado cf. Bulganbaatar y en marsupiales (Tabla 4, AD). La reducción en estos taxa, en relación a lo observado en cinodontes, se debe a la notable disminución del entepicóndilo. La condición de cf. Bulganbaatar es llamativa, mostrando una mayor reducción de los cóndilos radial y ulnar que en Didelphis (Sereno y McKenna, 1995, fig. 3e). Debe destacarse que la extremidad distal es notablemente amplia en el triconodonte Gobiconodon, correspondiendo aproximadamente a la mitad de la longitud humeral (Jenkins y Schaff, 1988), condición comparable a la de numerosos cinodontes.

La troclea en Gobiconodon y Didelphis es surcada, marcando una gran diferencia con la de cinodontes, 


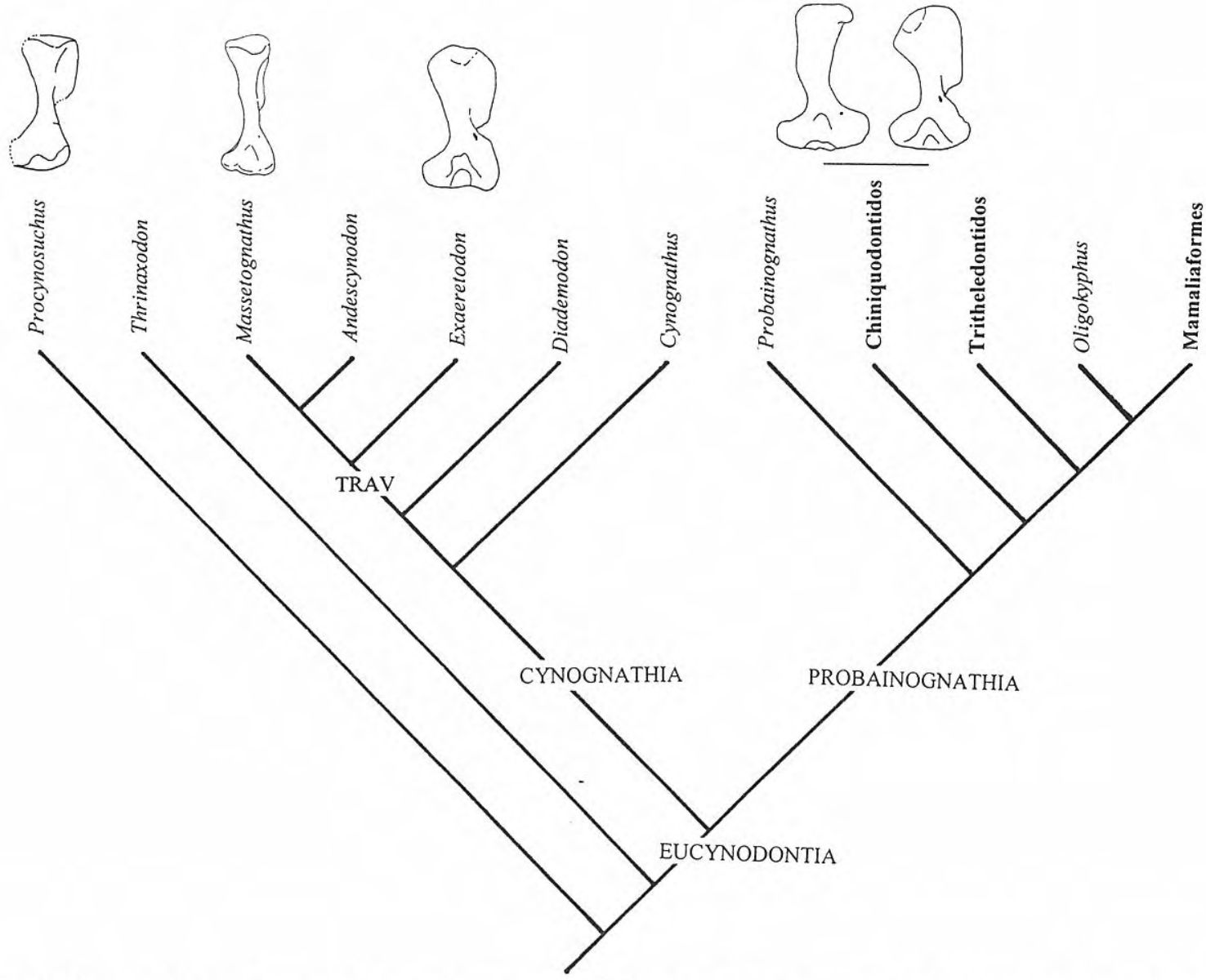

Figura 8. Cladograma de cinodontes no mamalianos, mostrando grupos que presentan el húmero con la cresta deltopectoral abierta. Nótese que en chiniquodóntidos (al igual que en traversodóntidos) este elemento presenta dos morfologías diferentes (ver en el texto). Relaciones filogenéticas según Abdala (1996b). No a escala.

morganucodontes, monotremas y multituberculados, donde esta es bulbosa (Jenkins y Schaff, 1988).

$\mathrm{El}$ foramen entepicondilar está muy desarrollado tanto en Gobiconodon como en Vincelestes, en ambos casos con una posición similar a la que presenta en cinodontes. En morganucodóntidos y Didelphis, dicho foramen es semejante al de cinodontes aunque está en una posición más distal en el hueso. En Vincelestes, el foramen se observa ensanchado, siendo su forma notablemente más circular (Rougier, 1993, fig. 85), mientras que en los multituberculados, el mismo tiene un desarrollo menor que el de cinodontes y restantes mamíferos no terios observados (Kielan-Jaworowska y Qi, 1990; Sereno y McKenna, 1995). Si bien la presencia del foramen entepicondilar es bien constante en mamíferos, es interesante remarcar la plasticidad del mismo en los placentarios dado que desaparece independientemente en distintos linajes, tales como úrsidos, équidos y cérvidos, entre otros.

En ninguno de los mamíferos examinados hay evidencias de la presencia de foramen ectepicondilar, condición semejante a la de Cynognathus, Probainognathus y tritilodontes. Los cinodontes traversodóntidos (por ejemplo ?Andescynodon, Pascualgnathus, Massetognathus y Exaeretodon) muestran una proyección en las proximidades del foramen ectepicondilar que puede interpretarse como esbozos de la eminencia intertuberal o tubérculo intermedio, estructura presente en Didelphis. Es interesante destacar que la extremidad distal del húmero de Macropus presenta una muesca profunda, delimitada por la proyección ectepicondilar en el lado externo (Lessertisseur y Saban, 1967), en una posición similar a donde se observa el foramen ectepicondilar de cinodontes. Dicha estructura también es conocida en otros marsupiales y en el edentado Cyclope, donde la proyección practicamente se cierra conformando nuevamente un foramen (Lessertisseur y Saban, 1967).

\section{CONCLUSIONES}

A partir de la descripción del húmero asociado al cráneo de Cynognathus se concluye que los húmeros asignados con dudas a cinognátidos (Watson, 1917; Jenkins, 1971) no pertenecen a este grupo, siendo morfológicamente similares al húmero de Diademodon.

El húmero de cinodontes se encuentra representado por dos patrones morfológicos diferenciados. Uno robusto y corto, con los extremos generalmente expandidos. Este patrón se observa en chiniquodóntidos y en el traversodóntido Exaeretodon. En el segundo patrón el húmero es más esbelto y las extremidades comparativamente menos expandidas transversalmente. 
Se registran variaciones entre cinodontes en la angulación de la cresta deltopectoral en relación al eje que pasa por la cabeza y la tuberosidad menor. La cresta conforma un ángulo muy abierto en formas filogenéticamente basales, como Procynosuchus y en representantes robustos de dos familias: traversodóntidos y chiniquodóntidos. Esta morfología diferencial en cuanto a la angulación de la cresta deltopectoral en formas robustas probablemente se relacione con una mayor área de inserción del músculo pectoral, que tendría más importancia en el sostén corporal en animales más grandes. La misma variación también se observa en dicinodontes, con la cresta deltopectoral más abierta en animales de mayor tamaño absoluto.

Las proporciones de la extremidad proximal y distal del húmero tienden a disminuir en dirección a los mamaliaformes (Tabla 4). El único cinodonte con proporciones similares a este último grupo en ambos índices es Probainognathus. El multituberculado ?Lambdopsalis presenta ambas extremidades muy expandidas, lo cual se relacionaría con un modo de vida fosorial (Kielan-Jaworowska y Qi, 1990). El extremo distal del húmero de Gobiconodon también presenta una notable expansión.

En los mamaliaformes (Fig. 8) existe un notable desarrollo de la cabeza humeral, mucho más proyectada dorsalmente. La cresta deltopectoral generalmente sobrepasa la mitad de la diáfisis humeral en el linaje cinodonte-mamalia, siendo solo menor en Eozostrodon y en Vincelestes. La mayor extensión de dicha cresta se verifica en el multituberculado ?Lambdopsalis y en Didelphis, aunque también hay cinodontes con la cresta muy extendida axialmente.

Las reducciones en las extremidades del húmero así como el mayor desarrollo de la cabeza a lo largo del linaje cinodontia-mamalia probablemente se relacionen con una posición de este elemento menos horizontal, más paralela al plano axial corporal y cada vez más próximo a posicionarse por debajo del cuerpo.

\section{AGRADECIMIENTOS}

Quisiera expresar mi agradecimiento a J. Powell, J. F. Bonaparte, A. Milner, P. Wellnhofer, A. Liebau, T. Kemp y R. Smith quienes me permitieron el acceso a material bajo su cuidado. Tom Kemp generosamente me facilitó el acceso a material postcraneano de chiniquodóntidos del Museum of Comparative Zoology de Harvard, en ese tiempo en Oxford. Fernando Lobo, Andrea Arcucci, Rafael Herbst realizaron sugerencias sobre el manuscrito inicial. James Hopson realizó sugerencias sobre el abstract. Jose F. Bonaparte y G. Rougier, como árbitros, realizaron observaciones y sugerencias de importancia para el resultado final de este trabajo. Susana Esteban incentivó en gran medida la realización de este estudio. La DAAD (Deutscher Akademischer fur Austaunsdienst), el Consejo Nacional de Investigación de Argentina (Conicet), la Universidad Nacional de Tucumán, el Conselho Nacional de Desenvolvimiento Científico e Tecnológico de Brasil
(CNPq) y la Pontificia Universidade Católica de Rio Grande do Sul financiaron los proyectos que permitieron la realizacion y culminación de este estudio.

\section{BIBLIOGRAFÍA}

Abdala, F. 1996a. Redescripción del cráneo y reconsideración de la validez de Cynognathus minor (Eucynodontia-Cynognathidae) del Triásico inferior de Mendoza. Ameghiniana, 33, 115-126.

Abdala, F. 1996b. Los Chiniquodontoideos (Synapsida, Cynodontia) sudamericanos. Tesis Doctoral, Facultad de Ciencias Naturales, Universidad Nacional de Tucumán, $398 \mathrm{pp}$.

Anderson, J.M. and Anderson, H. M. 1993. Terrestrial flora and fauna of the Gondwana Triassic: Part 1- Ocurrences. In: The nonmarine Triassic (Eds. S.G. Lucas and M. Morales). New Mexico Museum of Natural History and Science Bulletin, 3, 3-12.

Bonaparte, J.F. 1963. Descripción del esqueleto postcraneano de Exaeretodon (Cynodontia-Traversodontidae). Acta Geológica Lilloana, 4, 5-53.

Bonaparte, J.F. 1966. Una nueva "fauna" triásica de Argentina (Therapsida: Cynodontia Dicynodontia). Consideraciones filogenéticas y paleobiogeográficas. Ameghiniana, 4, 243-296.

Bonaparte, J.F. 1969. Cynognathus minor n. sp. (Therapsida-Cynodontia). Nueva evidencia de vinculación faunística afrosudamericana a principios del Triásico. Gondwana stratigraphy, I.U.G.S. Coloquio. Mar del Plata 1967, 273-281.

Bonaparte, J. F. 1978. El mesozoico de América del Sur y sus tetrápodos. Opera Lilloana, 26, 596 pp.

Brink, A. S. 1955. On the Cynognathidae. Palaeontologia Africana, 3, 47-55.

Broili, F. und Schröder, J. 1934. Zur Osteologie des Kopfes von Cynognathus. Sitzungsberichte der Bayer Akademie der Wissenschaften, Mathem-Naturwissenschaft. Abteil, 1934, 95-128.

Broili, F. und Schröder, J. 1935. Über den Schädel von Cynidiognathus Haughton. Sitzungsberichte der Bayer Akademie der Wissenschaften, Mathem-Naturwissenschaften. Abteil, 1935, 199-222.

Broom, R., 1932. The mammal-like reptiles of South Africa and the origin of mammals. H.G. Witherby, London. 376 pp.

Broom, R., 1948. A contribution to our knowledge of the vertebrates of the Karroo beds of South Africa. Transactions of the Royal Society of Edinburgh, 61, 577629.

Crompton, A.W. 1955. On some Triasic cynodonts from Tanganyika. Proccedings of the Zoological Society of London, 125, 617-669.

Cox, B., 1965. New Triassic dicynodonts from South America, their origins and relationships. Philosophical Transactions of the Royal Society of London, 753, 457516.

Gonzales Díaz, E. 1966. El hallazgo del infra?-mesotriásico continental en el sur del área pedemontana mendocina. Acta Geológica Lilloana, 8, 101-134. 
Gonzales Díaz, E. 1972. Descripción geológica de la hoja 27d, San Rafael, Provincia de Mendoza. Servicio Nacional Minero Geológico, Boletín 132, 127 pp.

Hancox, J. and Rubidge, B. 1996. The first specimen of the Mid-Triassic dicynodont Angonisaurus from the Karoo of South Africa: implications for the dating and biostratigraphy of the Cynognathus Assemblage Zone, Upper Beaufort Group. South African Journal of Science, 92, 391-392.

Hildebrand, M. 1988. Analysis of vertebrate structure. Third Edition, John Wiley \& Sons. 701 pp.

Hu, Y., Wang, Y., Luo, Z. and Li, C. 1997. A new symmetrodont mammal from China and its implications for mammalian evolution. Nature, 390, 137-142.

Huene, F. von 1935-42. Die fossilen Reptilien des Südamerikanischen Gondwanalandes. 2. Lieferung, Cynodontia. F. Heine, Tübingen, 93-159.

Jenkins, F. Jr. 1970a. The Chañares (Argentina) Triassic reptile fauna. VII. The postcranial skeleton of the traversodontid Massetognathus pascuali (TherapsidaCynodontia). Breviora, 352, 1-28.

Jenkins, F. Jr. 1970b. Limb movements in a monotreme (Tachyglossus aculeatus): a cineradiographic analysis. Science, 168, 1473-1475.

Jenkins, F. Jr. 1971. The postcranial skeleton of African cynodonts. Bulletin of the Peadbody Museum, 36, 216 pp.

Jenkins, F. Jr. 1973. The functional anatomy and evolution of the mammalian humero-ulnar articulation. American Journal of Anatomy, 137, 281-298.

Jenkins, F. Jr. and Parrington, F. 1976. The postcraneal skeleton of the Triassic mammals Eozostrodon, Megazostrodon and Erythrotherium. Philosophical Transactions of the Royal Society of London (B), 273, 387-431.

Jenkins, F. Jr. and Schaff, C. 1988. The Early Cretaceous mammal Gobiconodon (Mammalia, Triconodonta) from the Cloverly Formation in Montana. Journal of Vertebrate Paleontology, 8, 1-24.

Kemp, T. S. 1979. The primitive cynodont Procynosuchus: functional morphology of the skull and relationships. Philosophical Transactions of the Royal Society of London (B), 285, 73-122.

Kemp, T. S. 1980a. The primitive cynodont Procynosuchus: structure, function and evolution of the postcranial skeleton. Philosophical Transactions of the Royal Society of London (B), 288, 217-258.

Kemp, T. S. 1980b. Aspects of the structure and functional anatomy of the Middle Triassic cynodont Luangwa. Journal of Zoology of London, 191, 193-239.

Kemp, T. S. 1986. The skeleton of a baurioid therocephalian therapsid from the Lower Triassic (Lystrosaurus Zone) of South Africa. Journal of Vertebrate Paleontology, 6, 213-232.

Kielan-Jaworowska, Z. and Qi Tao. 1990. Fossorial adaptations of a Taeniolabidoid multituberculate mammal from the Eocene of China. Vertebrata Palasiatica, 28, 81-94.

King, G. M. 1993. Ecology and biogeography of Triassic non-mammalian therapsids. In: Evolution, ecology and biogeography of the Triassic reptiles (Eds. J. M. Mazin and G. Pinna). Paleontologia Lombarda della Societá Italiana di Scienze Naturali e del Museo Civico di Storia Naturale di Milano. Nuova Serie, 2, 73-82.

Kitching, J.W. 1977. The distribution of the Karroo vertebrate fauna. Bernard Price Institute for Palaeontological Research, Memoir 1, 1-131.

Kühne, W. 1956. The liassic therapsid Oligokyphus. British Museum (Natural History), London, 149 pp.

Lessertisseur, J. et Saban, R. 1967. Squelette appendiculaire. In: Traité de Zoologie (Dir. P. P. Grassé). Tomo 16, fasc. 1. Masson et Cie., Paris, 709-1078.

Ochev, V. G. and Shishkin, M. A. 1989. On the principles of global correlation of the continental Triassic on the tetrapods. Acata Paleontologica Polonica, 34, 149-173.

Romer, A. S. 1922. The locomotor apparatus of certain primitive and mammal-like reptiles. Bulletin of the American Museum of Natural History, 46, 517-606.

Romer, A. S. and Lewis, A. 1973. The Chañares (Argentina) triassic reptile fauna. Postcraneal materials of the cynodonts Probelesodon and Probainognathus. Breviora, 407, 1-26.

Rougier, G.W. 1993. Vincelestes neuquenianus Bonaparte (Mammalia, Theria) un primitivo mamífero del Cretácico Inferior de la Cuenca Neuquina. Tesis Doctoral, Universidad Nacional de Buenos Aires, 720 pp.

Rowe, T. 1988. Definition, diagnosis, and origin of Mammalia. Journal of Vertebrate Paleontology, 8, 241264.

Seeley, H., 1895a. Researches on the structure, organization, and classification of the fosil Reptilia. Pt. 9, Section 4. On the Gomphodontia. Philosophical Transactions of the Royal Society (B), 186, 1-57.

Seeley, H., 1895b. Researches on the structure, organization, and classification of the fosil Reptilia. Pt. 9, Section 5. On the skeleton in new cynodontia from the Karroo rocks. Philosophical Transactions of the Royal Society (B), 186, 59-148.

Sereno, P. C. and McKenna, M.C.. 1995. Cretaceous multituberculate skeleton and the early evolution of the mammalian shoulder girdle. Nature, 377, 144-147.

Sues, H. D. 1983. Advanced mammal-like reptiles from the Early Jurassic of Arizona. Philosophical Doctor Dissertation, Harvard University, 278 pp.

Sun, A. and Li, Y. 1985. The postcranial skeleton of the late tritylodont Bienotheroides. Vertebrata Palasiatica, 23, 135-151.

Walker, W. F. Jr. 1987. Functional anatomy of the vertebrates. An evolutionary perspective. Saunders College Publishing, $781 \mathrm{pp}$.

Watson, D. M. 1917. The evolution of the tetrapod shoulder girdle and forelimb. Journal of Anatomy, 52, 1-63.

Young, C.C. 1947. Mammal-like reptiles from Lufeng Yunnan, China. Procedings of the Zoological Society of London, 177, 537-597. 